\title{
Hypersound Absorption of Acoustic Phonons in a Degenerate Carbon Nanotube
}

\author{
K. A. Dompreh', N. G. Mensah², S. Y. Mensah1', S. S. Abukari1, F. Sam¹, R. Edziah1 \\ ${ }^{1}$ Department of Physics, College of Agriculture and Natural Sciences, U.C.C, Cape Coast, Ghana \\ ${ }^{2}$ Department of Mathematics, College of Agriculture and Natural Sciences, U.C.C, Cape Coast, Ghana \\ Email: kwadwo.dompreh@ucc.edu.gh
}

Received 24 June 2015; accepted 27 July 2015; published 31 July 2015

Copyright (C) 2015 by authors and Scientific Research Publishing Inc.

This work is licensed under the Creative Commons Attribution International License (CC BY).

http://creativecommons.org/licenses/by/4.0/

(c) (i) Open Access

\section{Abstract}

Absorption of acoustic phonons was studied in degenerate Carbon Nanotube (i.e. where the electrons are found close to the Fermi level). The calculation of the hypersound absorption coefficient $(\Gamma)$ was done in the regime where $q l \gg l(q$ is the acoustic phonon number and $l$ is the electron mean free path). At $T=10 \mathrm{~K}$ and $\theta>0$ ( $\theta$ being scattering angle), the dependence of $\Gamma$ on acoustic wave number $(q)$, frequency $\left(\omega_{q}\right)$, and $\gamma=1-\frac{V_{D}}{V_{s}},\left(V_{s}\right.$ and $V_{D}$ being the speed of sound and the drift velocity respectively) were analysed numerically at $n=0, \pm 1, \pm 2$ (where $n$ is an integer) and presented graphically. It was observed that when $\gamma<0$, the maximum amplification was attained at $V_{D}=1.1 V_{s}$ which occurred at $E=51.7 \mathrm{~V} \cdot \mathrm{cm}^{-1}$. In the second harmonics, $(n= \pm 2)$, the absorption obtained was compared to experimental measurement of acoustoelectric current via the Weinreich relation and the results qualitatively agreed with each other.

\section{Keywords}

Carbon Nanotube, Hyperound, Absorption, Acoustoelectric

\section{Introduction}

Carbon Nanotubes (CNT) are graphite sheets seamlessly wrapped into cylinders characterised by a chiral index $(m, n)$, with $m$ and $n$ being two integers, which specify the carbon nanotube uniquely [1]. This has recently attracted lot of interest for use in many semiconductor devices due to its remarkable electrical, mechanical, and thermal properties which are mainly attributed to its unusual band structures [2]. The $\pi$-bonding and anti-bond- 
ing $\left(\pi^{*}\right)$ energy band of CNT crosses at the Fermi level in a linear manner [3] [4]. In the linear regime, electron-phonon interactions in CNT at low temperatures lead to the emission of large number of coherent acoustic phonons. Studies of the effect of phonons on thermal transport [5], on Raman scattering [6] and on electrical transport [7] in CNT are active areas of research. Also, the speed of electrons in the linear region is extremely high. This makes CNT a good candidate for application of high frequency electronic systems such as field effect transistors (FET's) [8], single electron memories [9] and chemical sensors [10]-[13]. Another important investigation in the linear regime is interaction of acoustic phonons with drift charges in CNT. It is well known that when acoustic phonons interact with charge carriers, it is accompanied by energy and momentum exchange which gives rise to the following effects: Absorption (Amplification) of acoustic phonons [14]-[17]; Acoustoelectric Effect (AE) [18]-[21]; Acoustomagnetoelectric Effect (AME) [22]; Acoustothermal Effect [23] and Acoustomagnetothermal Effect [24] [25]. The idea of acoustic wave amplification in bulk material was theoretically predicted by Tolpygo and Uritskii (1956) [26], and Weinreich [27] and in N-Ge by Pomerantz [28]. In Superlattices, the effect of hypersound absorption/amplification was extensively studied by Mensah et al. [29][33], Vyazovsky et al. [34], Bau et al. [35], while Shmelev and Zung [36] calculated the absorption coefficient and renormalization of the short-wave sound velocity. Azizyan [37] calculated the absorption coefficient in a quantized electric field. Furthermore, Acoustic wave absorption/amplification in Graphenes [38]-[40], Rectangular quantum wires [41], and quantum dots [42] have all received attention. On the concept of Acoustoelectric Effect (AE) in bulk [43] [44] and low-dimensional materials, lots of researches have been comprehensively done both theoretically and experimentally. Acoustoelectric effect in CNT's is now receiving attention with few experimental works done on it. Ebbecke et al. [45] studied the AE current transport in a single walled CNT, whilst Reulet et al. [46] studied AE in CNT. But in all these researches, there are no theoretically studies of AE in CNT. In this paper, the absorption (amplification) of hypersound in CNT in the regime $q l \gg$ ( $q$ is the acoustic wave number and $l$ is the electron mean free path) is considered where the acoustic wave is considered as a flow of monochromatic phonons of frequency $\left(\omega_{q}\right)$.

It is worth noting that the mechanism of absorption (amplification) is due to Cerenkov effect. For practical use of the Cerenkov acoustic-phonon emission, the material must have high drift velocities and large densities of electrons. Carbon Nanotubes (CNT) has electron mobility of $10^{5} \mathrm{~cm}^{2} / \mathrm{V} \cdot \mathrm{s}$ at room temperature. At low temperature $(T=10 \mathrm{~K})$, CNT exhibit good AE effect, which indicates that Cerenkov emission can take place in it [47]. The paper is organised as follows: In Section 2, the kinetic theory based on the linear approximation for the phonon distribution function is setup, where the rate of growth of the phonon distribution is deduced and the absorption coefficient $(\Gamma)$ is obtained. In Section 3, the final equation is analysed numerically in a graphical form at various harmonics where the absorption obtained are related to the acoustoelectric current via the Wienrich relation. Lastly the conclusion is presented in Section 4.

\section{Theory}

We will proceed following the works of [48] [49] where the kinetic equation for the phonon distribution is given as

$$
\begin{aligned}
\frac{\partial N_{q}}{\partial t}= & \frac{2 \pi}{\hbar} \sum_{p}\left|C_{q}\right|^{2}\left\{\left[N_{q}(t)+1\right] f_{p}\left(1-f_{p^{\prime}}\right) \delta\left(\varepsilon_{p^{\prime}}-\varepsilon_{p}+\hbar \omega_{q}\right)\right. \\
& \left.-N_{q}(t) f_{p^{\prime}}\left(1-f_{p}\right) \delta\left(\varepsilon_{p^{\prime}}-\varepsilon_{p}-\hbar \omega_{q}\right)\right\}
\end{aligned}
$$

where $N_{q}(t)$ represents the number of phonons with wave vector $\boldsymbol{q}$ at time $t$. The factor $N_{q}+1$ accounts for the presence of $N_{q}$ phonons in the system when the additional phonon is emitted. The $f_{p}\left(1-f_{p}\right)$ represents the probability that the initial $\boldsymbol{p}$ state is occupied and the final electron state $\boldsymbol{p}^{\prime}$ is empty whilst the factor $N_{q} f_{p^{\prime}}\left(1-f_{p}\right)$ is that of the boson and fermion statistics. The unperturbed electron distribution function is given by the shifted Fermi-Dirac function as

$$
f_{p}=\left[\exp \left(-\beta\left(\varepsilon\left(\boldsymbol{p}-m v_{D}\right)-\mu\right)\right)\right]^{-1}
$$

where $f_{\boldsymbol{p}}$ is the Fermi-Dirac equilibrium function, with $\mu$ being the chemical potential, $\boldsymbol{p}$ is momentum of the electron, $\beta=1 / k T, k$ is the Boltzmann constant and $V_{D}$ is the net drift velocity relative to the ion lattice site. In a more convenient form, Equation (1) can be written as 


$$
\begin{aligned}
\frac{\partial N_{\boldsymbol{q}}(t)}{\partial t}= & 2 \pi\left|C_{q}\right|^{2}\left[\frac{N_{\boldsymbol{q}}(t)+1}{1-\exp \left(-\beta\left(\hbar \omega_{\boldsymbol{q}}-\hbar \boldsymbol{q} \cdot V_{D}\right)\right)}+\frac{N_{\boldsymbol{q}}}{1-\exp \left(-\beta\left(\hbar \omega_{\bar{q}}-\hbar \boldsymbol{q} \cdot V_{D}\right)\right)}\right] \\
& \times \sum_{p}\left(f_{p}-f_{p^{\prime}}\right) \delta\left(\varepsilon_{\boldsymbol{p}^{\prime}}-\varepsilon_{\boldsymbol{p}}+\hbar \omega_{\boldsymbol{q}}\right)
\end{aligned}
$$

To simplify Equation (3), the following were utilised

$$
\begin{gathered}
Q=\sum_{p} \frac{f_{p}-f_{p^{\prime}}}{\varepsilon_{p}-\varepsilon_{p^{\prime}}-\hbar \omega_{q}-i \delta} \\
f_{p}=\left[\exp \left(-\beta\left(\varepsilon_{p}-\mu\right)\right)+1\right]^{-1}
\end{gathered}
$$

Given that

$$
\Gamma_{q}=-2\left|C_{q}\right|^{2} \operatorname{Im} Q\left(\hbar \boldsymbol{q}, \hbar \omega_{q}-\hbar \boldsymbol{q} \cdot V_{D}\right)
$$

the phonon generation rate simplifies to

$$
\Gamma_{q}=2 \pi\left|C_{q}\right|^{2} \sum_{p}\left(f_{p}-f_{p^{\prime}}\right) \delta\left(\varepsilon_{p}-\varepsilon_{p^{\prime}}-\left(\hbar \omega_{q}-\hbar \boldsymbol{q} \cdot V_{D}\right)\right)
$$

In Equation (7), $f_{p}>f_{p^{\prime}}$ if $\varepsilon_{p}<\varepsilon_{p^{\prime}}$. When $\hbar \omega_{q}-\hbar \boldsymbol{q} \cdot V_{D}>0$, the system would return to its equilibrium configuration when perturbed where

$$
N_{\boldsymbol{q}}^{0}=\left[\exp \left(-\beta\left(\hbar \omega_{\boldsymbol{q}}-\hbar \boldsymbol{q} \cdot V_{D}\right)-1\right)\right]^{-1}
$$

But $\hbar \omega_{q}-\hbar \boldsymbol{q} \cdot V_{D}<0$ leads to the Cerenkov condition of phonon instability (amplification). The linear energy dispersion $\varepsilon(\boldsymbol{p})$ relation for the CNT is given as [50]

$$
\varepsilon(\boldsymbol{p})=\varepsilon_{0} \pm \frac{\sqrt{3}}{2 \hbar} \gamma_{0} b\left(\boldsymbol{p}-\boldsymbol{p}_{0}\right)
$$

The $\varepsilon_{0}$ is the electron energy in the Brillouin zone at momentum $p_{0}, b$ is the lattice constant, $\gamma_{0}$ is the tight binding overlap integral $\left(\gamma_{0}=2.54 \mathrm{eV}\right)$. The \pm sign indicates that in the vicinity of the tangent point, the bands exhibit mirror symmetry with respect to each point. The phonon and the electric field are directed along the CNT axis, therefore $\boldsymbol{p}^{\prime}=(\boldsymbol{p}+\hbar \boldsymbol{q}) \cos (\theta)$. Where $\theta$ is the scattering angle. At low temperature, the $k T \ll 1$, Equation (5) reduces to

$$
f_{p}=\exp (-\beta(\varepsilon(p)-\mu))
$$

Inserting Equation (8) and Equation (9) into Equation (7), and after some cumbersome calculations yield

$$
\begin{array}{r}
\Gamma=\frac{4 \hbar \pi\left|C_{\boldsymbol{q}}\right|^{2} \exp \left(-\beta\left(\varepsilon_{0}-\chi \boldsymbol{p}_{0}\right)\right)}{\gamma_{0} b \sqrt{3}(1-\cos (\theta))}\{\exp (-\beta \chi(\eta+\hbar \boldsymbol{q}) \cos (\theta))-\exp (-\beta \chi \eta)\} \\
\text { where } \chi=\sqrt{3} \gamma_{0} b / 2 \hbar \text {, and } \eta=\frac{2 \hbar^{2} \omega_{\boldsymbol{q}}\left(1-\frac{V_{D}}{V_{s}}\right)+\gamma_{0} b \sqrt{3} \hbar \boldsymbol{q} \cos (\theta)}{\gamma_{0} b \sqrt{3}(1-\cos (\theta))}
\end{array}
$$

\section{Numerical Analysis}

Considering the finite electron concentration, the matrix element can be modified as

$$
\left|C_{q}\right|^{2} \rightarrow \frac{\left|C_{q}\right|^{2}}{\left|\aleph^{(e l)}(\boldsymbol{q})\right|^{2}}
$$


V 
K. A. Dompreh et al.

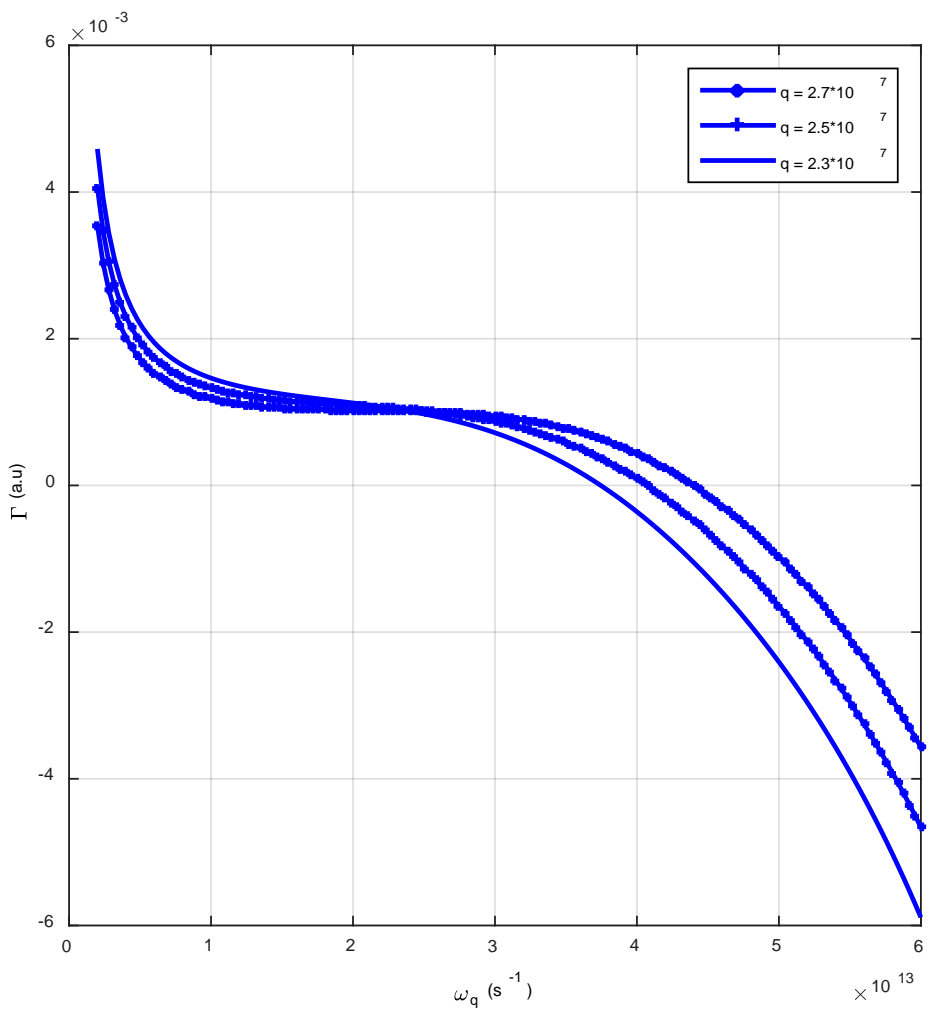

(b)

Figure 1. (a) Dependence of $\Gamma$ on $q$ for varying $\omega_{q}$ at $V_{D}=1.2 V_{s}$; (b) $\Gamma$ on $\omega_{q}$ for varying $q$ at $V_{D}=1.2 V_{s}$.

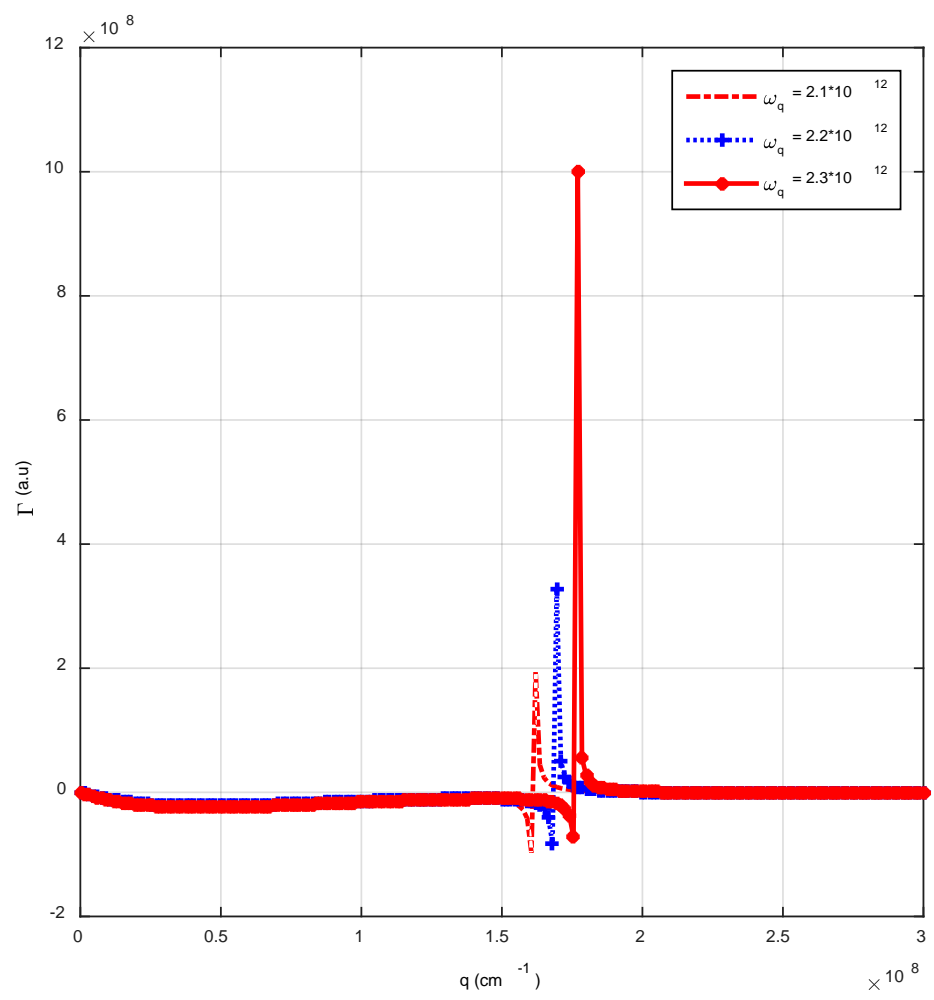

(a)

66 


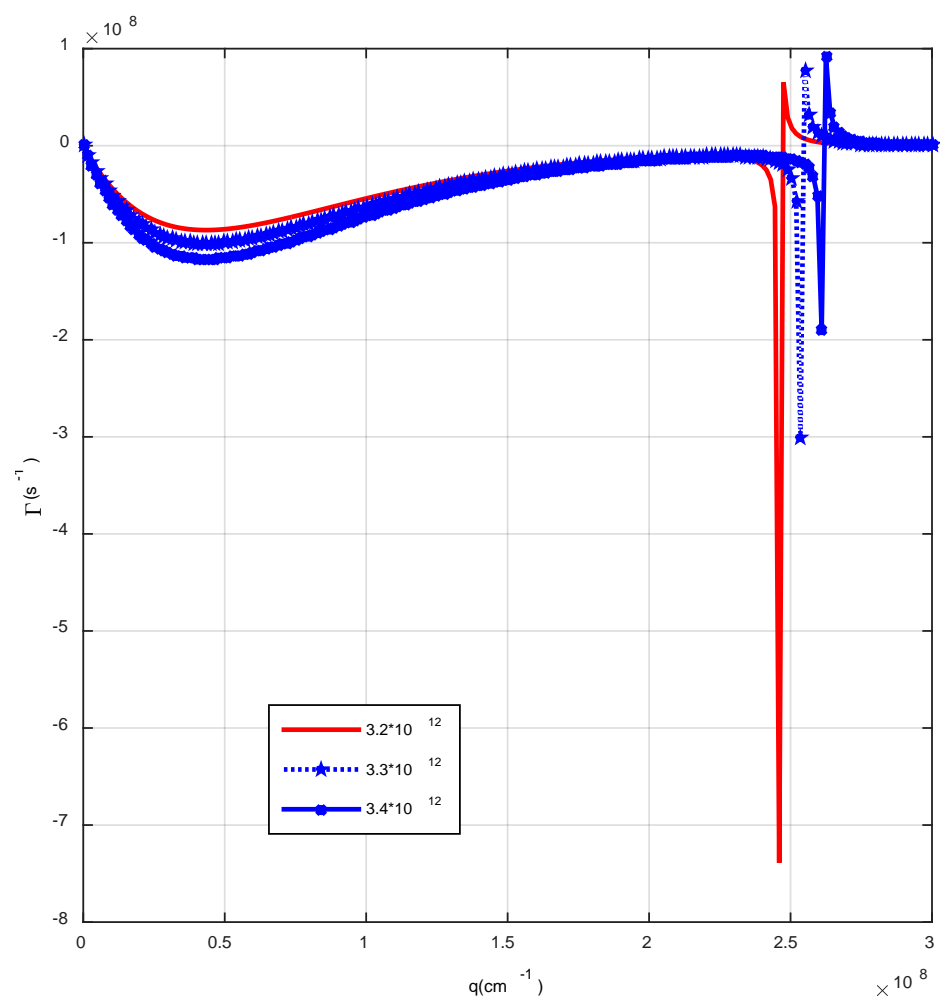

(b)

Figure 2. Dependence of $\Gamma$ on $\boldsymbol{q}$ at $V_{D}=1.2 V_{s}$ showing (a) Absorption exceeds Amplification; (b) Amplification exceeds Absorption.

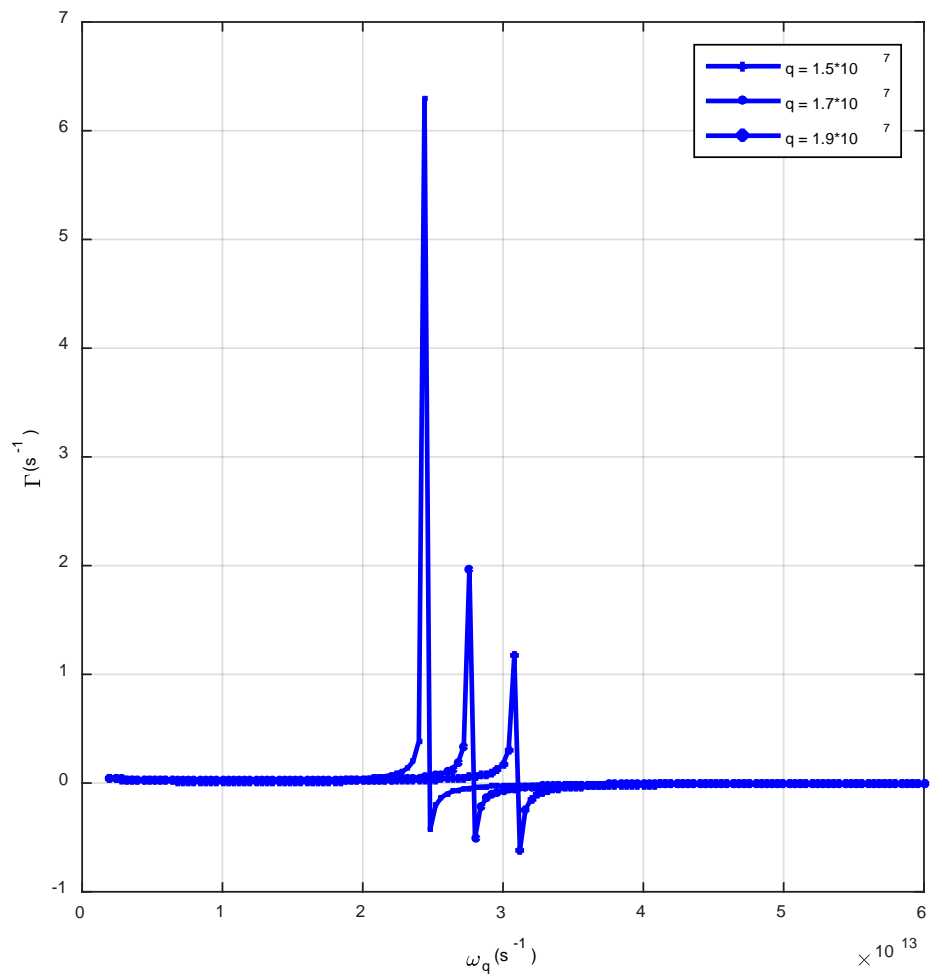

(a) 


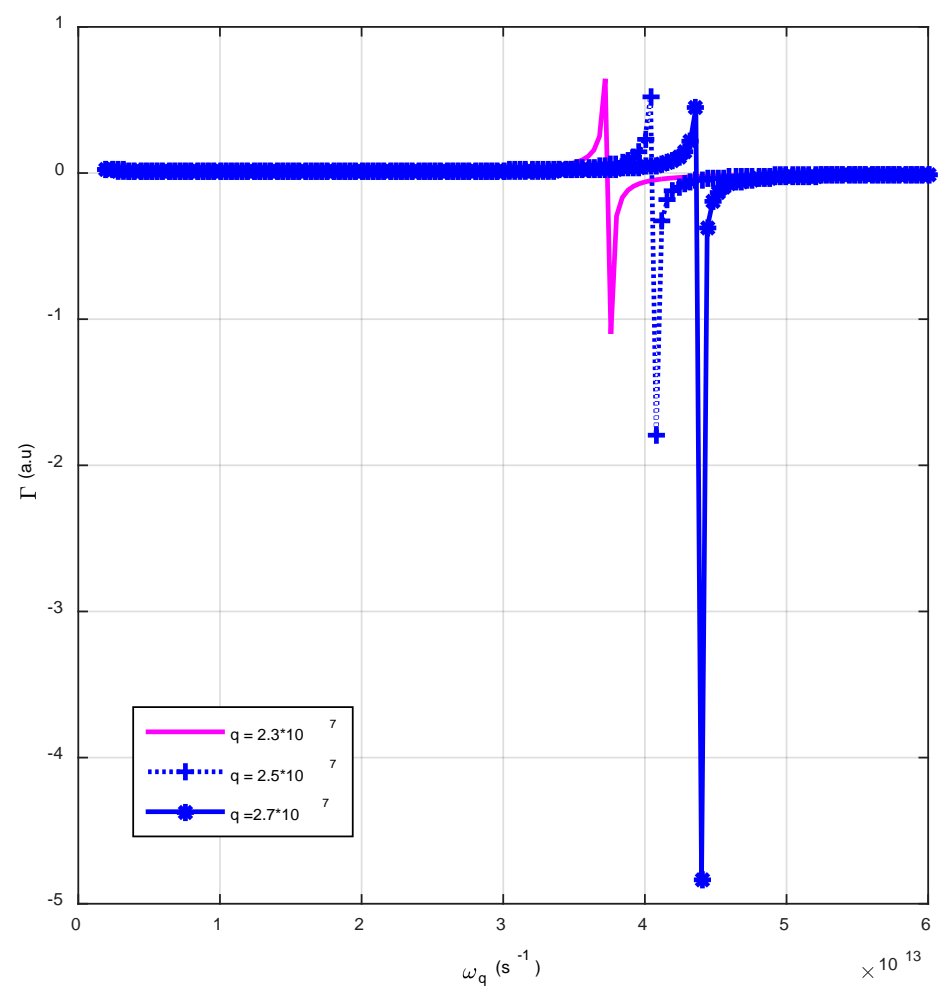

(b)

Figure 3. Dependence of $\Gamma$ on $\omega_{q}$ (a) Absorption exceeds Amplification; (b) Amplification exceeds Absorption.

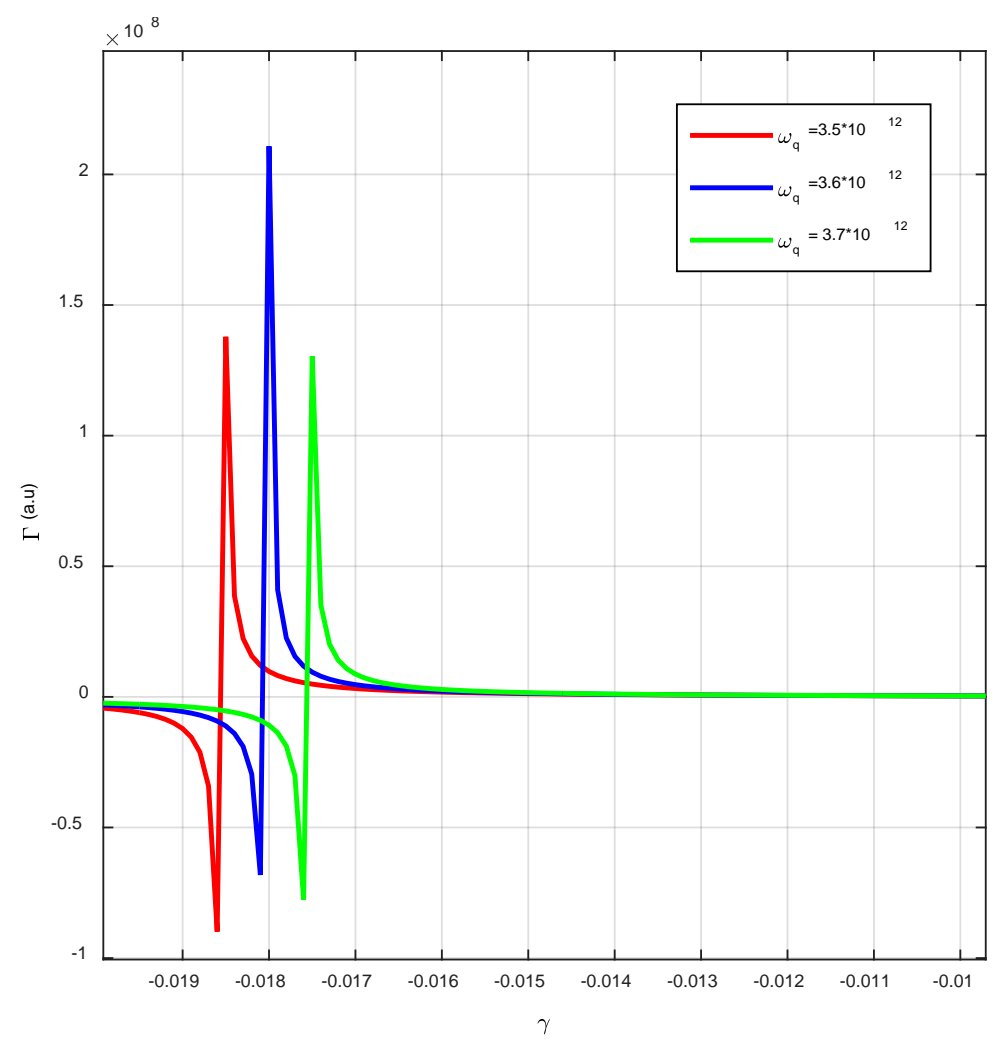

(a) 


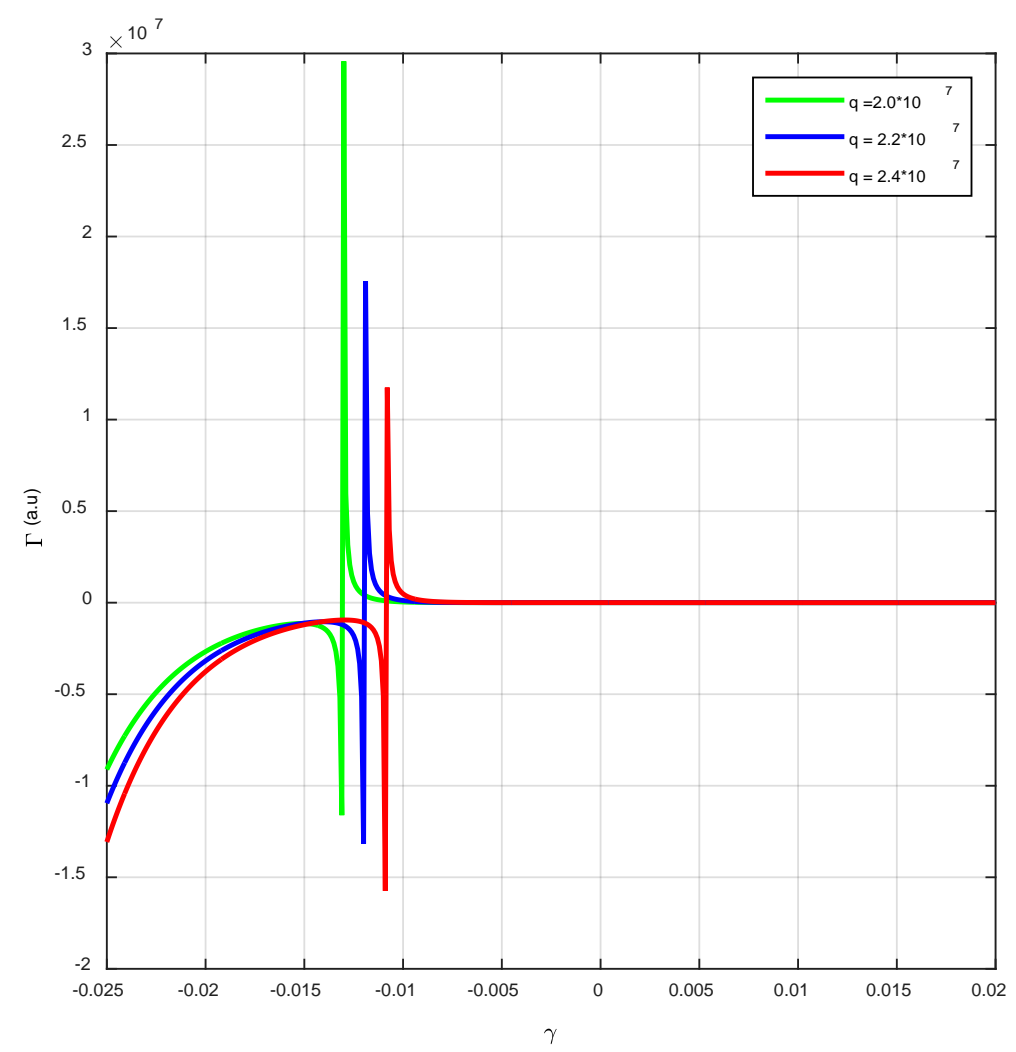

(b)

Figure 4. Dependence of $\Gamma$ on $\gamma$ at $\theta=80$ (a) By increasing $\omega_{q}$; (b) By increasing $q$.

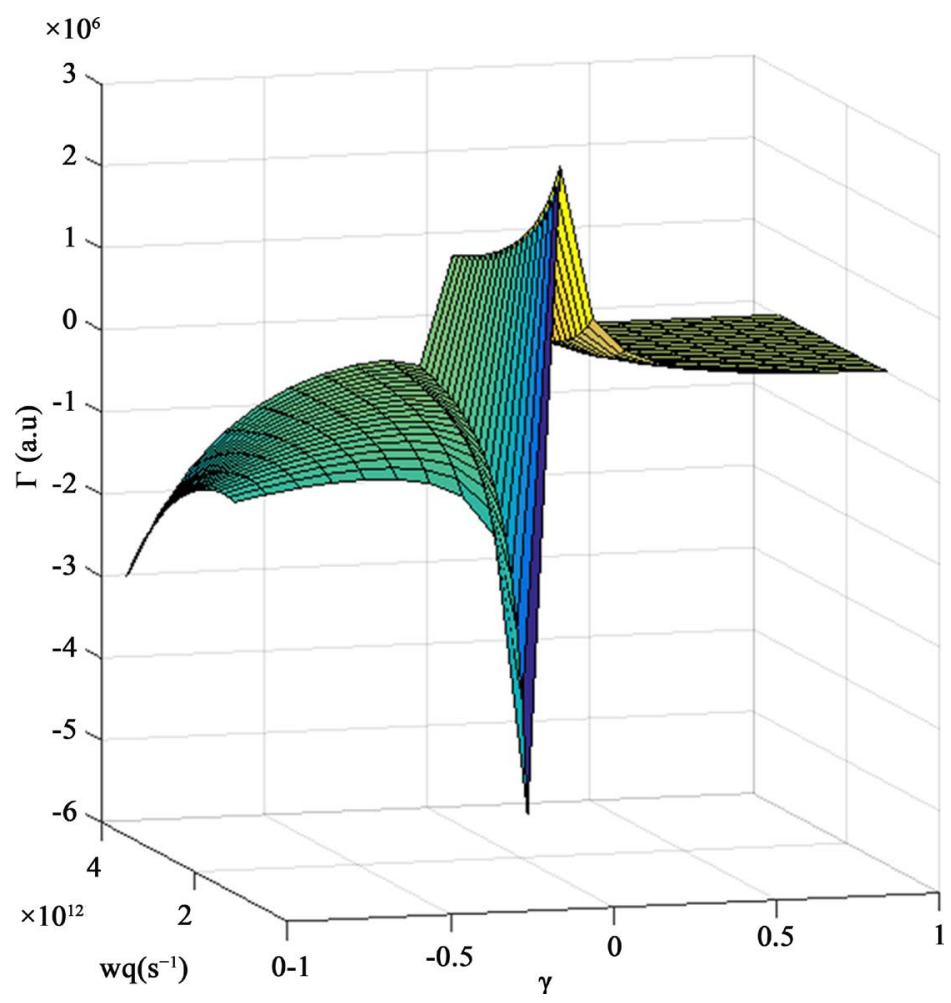

(a) 


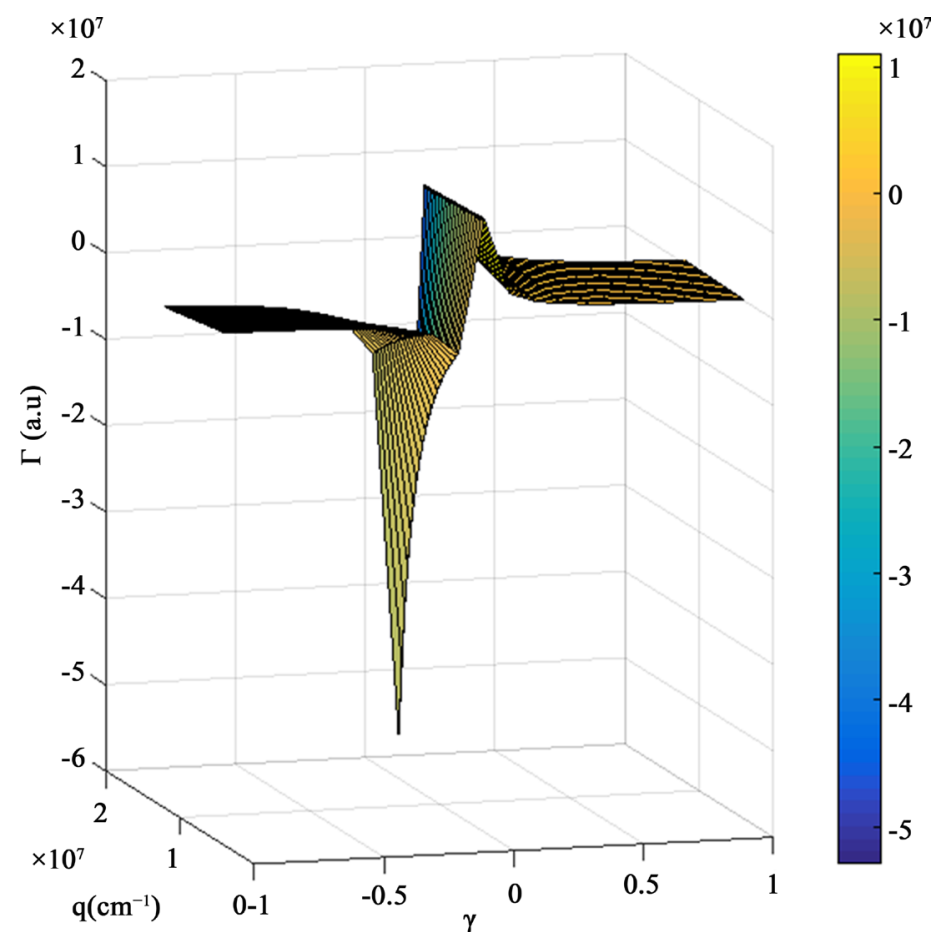

(b)

Figure 5. (a) Dependence of $\Gamma$ on $\omega_{q}$ and $\gamma$ and (b) Dependence of $\Gamma$ on $\boldsymbol{q}$ and $\gamma$.

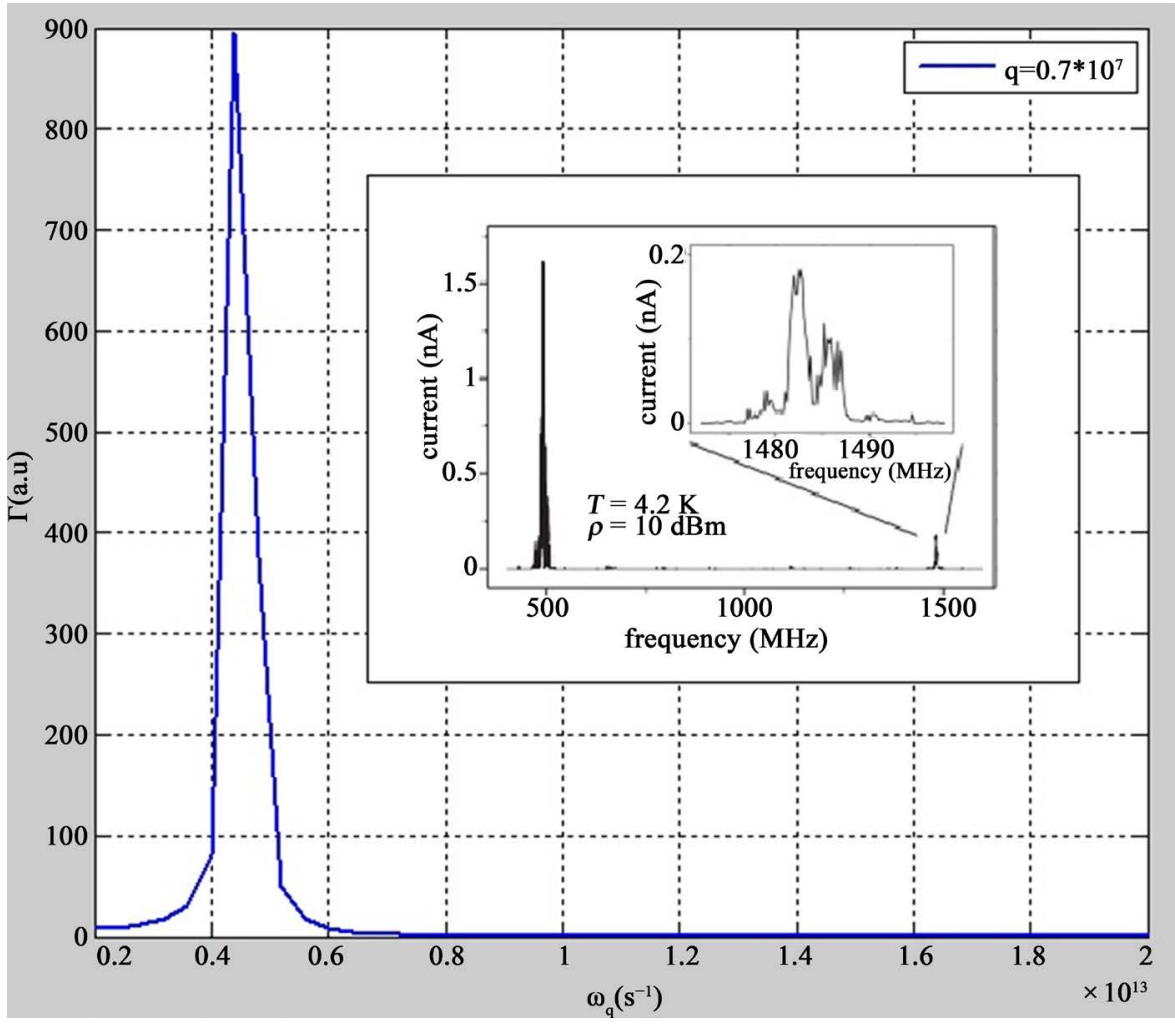

Figure 6. Second harmonic graph of the dependence of $\Gamma$ on $\omega_{q}$. Insert shows the experimental graph for acoustoelectric current versus frequency [45]. 


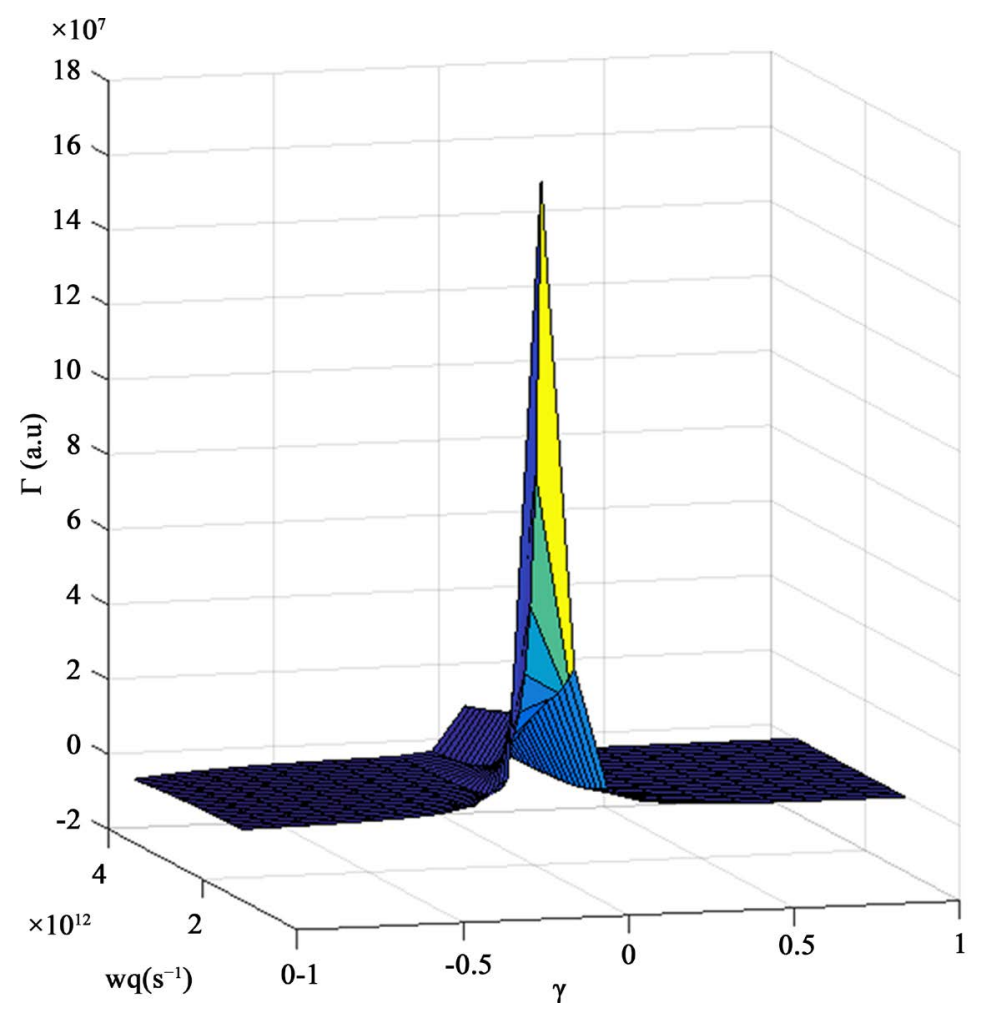

(a)

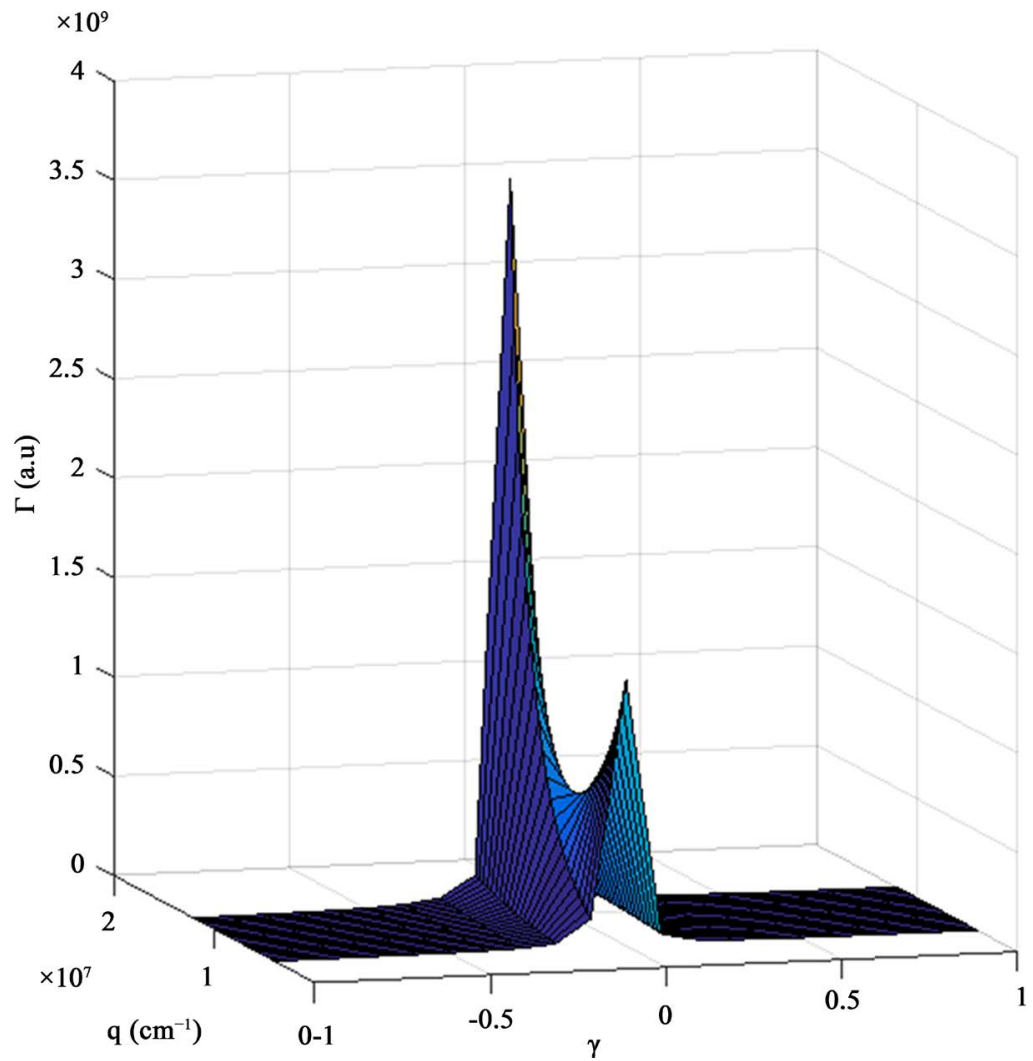

(b)

Figure 7. (a) Dependence of $\Gamma$ on $\boldsymbol{q}$ and $\gamma$ and (b) Dependence of $\Gamma$ on $\omega_{q}$ and $\gamma$. 
For $n= \pm 2$ (Second harmonics), the dependence of the absorption coefficient $\Gamma$ on $\omega_{q}$ is presented in 2D and 3D form as shown in Figure 6 and Figure 7. In Figure 6, an absorption graph was obtained. The insert shown is an experimental results obtained for the Acoustoelectric current in Single walled Carbon Nanotube. Figure 7 (Figure 7(a) and Figure 7 (b)) is the 3D representation of the absorption in second harmonics.

From Weinreich relation [33], the absorption coefficient is directly related to the acoustoelectric current, therefore from Figure 6, the results obtained for the absorption coefficient qualitatively agrees with the experimental results presented (see insert). In the 3D graphs, the maximum amplification and absorption occurs at $\gamma=-0.1$ which is equivalent to $V_{D}=1.1 V_{s}$, with the electric field $E=\frac{V_{D}}{\mu}$ gives $E=51.7 \mathrm{~V} / \mathrm{cm}$.

\section{Conclusion}

The expression for Hypersound Absorption of acoustic phonons in a degenerate Carbon Nanotube (CNT) was deduced theoretically and graphically as presented. In this work, the acoustic waves were considered to be a flow of monochromatic phonons in the short wave region $(q l \gg 1)$. The general expression obtained was analysed numerically for $n=0, \pm 1, \pm 2$ (where $n$ is an integer). From the graphs, at certain values of $\omega_{q}$ and $\boldsymbol{q}$, an Amplification was observed to exceed Absorption or vice-versa. For $\gamma<0$, the maximum Amplification was observed at $V_{D}=1.1 V_{s}$ which gave us a field of $E=51.7 \mathrm{~V} \cdot \mathrm{cm}^{-1}$. This field was far lower than that observed in superlattice and homogeneous semiconductors permitting the CNT to be a suitable material for hypersound generator (SASER). A similar expression can be seen in the works of Nunes and Fonseca.

What is very interesting to our work is the qualitative agreement of the absorption graph to an experimental graph resulting from an acoustoelectric current via the Weinriech relation.

\section{References}

[1] Iijima, S. (1991) Helical Microtubules of Graphitic Carbon. Nature, 354, 56-58. http://dx.doi.org/10.1038/354056a0

[2] Dresselhaus, M.S., Dresselhaus, G. and Jorio, A. (2004) Unusual Properties and Structure of Carbon Nannotubes. Annual Review of Materials Research, 34, 247-278. http://dx.doi.org/10.1146/annurev.matsci.34.040203.114607

[3] Saito, R., Dresselhaus, G. and Dresselhaus, M. (1998) Physical Properties of Carbon Nanotubes. Imperial College Press, London.

[4] Dresselhaus, M.S. and Eklund, P.C. (2000) Phonons in Carbon Nanotubes. Advances in Physics, 49, 705-814. http://dx.doi.org/10.1080/000187300413184

[5] LeRoy, B.J., Lemay, S.G., Kong, J. and Dekker, C. (2004) Electrical Generation and Absorption of Phonons in Carbon Nanotubes. Nature, 432, 371-374. http://dx.doi.org/10.1038/nature03046

[6] Kim, J.-H., Nugraha, A.R.T., Booshehri, L.G., Haroz, E.H., Sato, K., Sanders, G.D., Yee, K.-J., Lim, Y.-S., Stanton, C.J., Saito, R. and Kono, J. (2013) Coherent Phonons in Carbon Nanotubes and Grapheme. Chemical Physics, 413, 5580. http://dx.doi.org/10.1016/j.chemphys.2012.09.017

[7] Small, J.P., Shi, L. and Kim, P. (2003) Mesoscopic Thermal and Thermoelectric Measurements of Individual Carbon Nanotubes. Solid State Communications, 127, 181-186. http://dx.doi.org/10.1016/S0038-1098(03)00341-7

[8] Kane, C.L., Thess, A., Mele, E.J., Smalley, R.E., Lee, R.S., Fischer, J.E., Verschueren, A.R.M., Petit, P., Tans, S.J., Dai, H. and Dekker, C. (1998) Temperature-Dependent Resistivity of Single-Wall Carbon Nanotubes. Europhysics Letters, 41, 683-688. http://dx.doi.org/10.1209/epl/i1998-00214-6

[9] Sanders, G.D., Nugraha, A.R.T., Sato, K., Kim, J.-H., Kono, J., Saito, R. and Stanton, C.J. (2013) Theory of Coherent Phonons in Carbon Nanotubes and Graphene Nanoribbons. Journal of Physics: Condensed Matter, 25, Article ID: 144201. http://dx.doi.org/10.1088/0953-8984/25/14/144201

[10] Yao, Z., Kane, C.L. and Dekker, C. (2000) High-Field Electrical Transport in Single-Wall Carbon Nanotubes. Physical Review Letters, 84, Article ID: 2941. http://dx.doi.org/10.1103/PhysRevLett.84.2941

[11] Dekker, C., Tans, S.J. and Verschueren, A.R.M. (1998) Room-Temperature Transistor Based on a Single Carbon Nanotube. Nature, 393, 49-52. http://dx.doi.org/10.1038/29954

[12] Kim, B.M., Chen, Y.-F. and Fuhrer, M.S. (2002) Single-Electron Detection and Memory Using a Single Carbon Nanotube Defect. The Exciting World of Nanocages and Nanotubes, 12, 541-548.

[13] Peng, S., O’Keeffe, J., Wei, C., Cho, K., Kong, J. and Chen, R. (2001) Carbon Nanotube Chemical and Mechanical Sensors. Proceedings of the 3rd International Workshop on SHM, Stanford, 12-14 September 2001.

[14] Mensah, S.Y., Allotey, F.K.A., Mensah, N.G. and Nkrumah, G. (2001) Differential Thermopower of a CNT Chiral 
Carbon Nanotube. Journal of Physics: Condensed Matter, 13, 5653-5662.

http://dx.doi.org/10.1088/0953-8984/13/24/310

[15] Mensah, S.Y., Allotey, F.K.A., Mensah, N.G. and Nkrumah, G. (2003) Giant Electrical Power Factor in Single-Walled Chiral Carbon Nanotube. Superlattices and Microstructures, 33, 173-180. http://dx.doi.org/10.1016/S0749-6036(03)00070-3

[16] Mensah, S.Y., Twum, A., Mensah, N.G., Dompreh, K.A., Abukari, S.S. and Nkrumah-Buandoh, G. (2011) Effect of Laser on Thermopower of Chiral Carbon Nanotube. http://arxiv.org/abs/1104.1913

[17] Mensah, N.G., Nkrumah, G., Mensah, S.Y. and Allotey, F.K.A. (2004) Temperature Dependence of the Thermal Conductivity in Chiral Carbon Nanotubes. Physics Letters A, 329, 369-378. http://dx.doi.org/10.1016/j.physleta.2004.06.100

[18] Shmelev, G.M., Anh, N.Q., Tsurkan, G.I. and Mensah, S.Y. (1984) "Currentless” Amplification of Hypersound in Planar Configuration by Inelastic Scattering of Electrons. Physica Status Solidi (b), 121, 209-213.

[19] Mensah, S.Y., Allotey, F.K.A. and Adjepong, S.K. (1994) The Effect of a High-Frequency Electric Field on Hypersound Amplification in a Superlattice. Journal of Physics: Condensed Matter, 6, 3479-3485. http://dx.doi.org/10.1088/0953-8984/6/19/003

[20] Parmenter, R.H. (1953) The Acousto-Electric Effect. Physical Review, 89, 990-998. http://dx.doi.org/10.1103/PhysRev.89.990

[21] Maao, F.A. and Galperin, Y. (1997) Acoustoelectric Effects in Quantum Constrictions. Physical Review B, 56, 40284036. http://dx.doi.org/10.1103/PhysRevB.56.4028

[22] Grinberg, A.A. and Kramer, N.I. (1965) Acoustomagnetic Effect in Piezoelectric Semiconductors. Soviet Physics Doklady, 9, 552.

[23] Yamada, T. (1965) Acoustomagnetoelectric Effect in Bismuth. Journal of the Physical Society of Japan, 20, 14241437. http://dx.doi.org/10.1143/JPSJ.20.1424

[24] Margulis, A.D. and Margulis, V.A. (1994) The Quantum Acoustomagnetoelectric Effect Due to Rayleigh Sound Waves. Journal of Physics: Condensed Matter, 6, 6139-6150. http://dx.doi.org/10.1088/0953-8984/6/31/013

[25] Mensah, S.Y. and Kangah, G.K. (1991) Amplification of Acoustic Waves Due to an External Temperature Gradient in Superlattices. Journal of Physics: Condensed Matter, 3, 4105-4107. http://dx.doi.org/10.1088/0953-8984/3/22/020

[26] Tolpygo, K.B. and Uritskii, Z.I. (1956) Article title. Zh. Eksp. Teor. Fiz., 30, 929.

[27] Weinreich, G. (1956) Acoustodynamic Effects in Semiconductors. Physical Review, 104, 321-324. http://dx.doi.org/10.1103/PhysRev.104.321

[28] Pomerantz, M. (1964) Amplification of Microwave Phonons in Germanium. Physical Review Letters, 13, 308-310. http://dx.doi.org/10.1103/PhysRevLett.13.308

[29] Mensah, S.Y., Allotey, F.K.A. and Adjepong, S.K. (1996) Acoustomagnetoelectric Effect in a Superlattice. Journal of Physics: Condensed Matter, 8, 1235-1239. http://dx.doi.org/10.1088/0953-8984/8/9/014

[30] Mensah, S.Y., Allotey, F.K.A. and Adjepong, S.K. (1994) Acoustoelectric Effect in Semiconductor Superlattice. Journal of Physics: Condensed Matter, 6, 6783-6787.

[31] Mensah, S.Y., Allotey, F.K.A. and Mensah, N.G. (2000) Nonlinear Acoustoelectric Effect in a Semiconductor Superlattice. Journal of Physics: Condensed Matter, 12, 5225-5232. http://dx.doi.org/10.1088/0953-8984/12/24/313

[32] Mensah, S.Y., Allotey, F.K.A., Mensah, N.G., Akrobotu, H. and Nkrumah, G. (2005) The Influence of External Electric Field on Acoustoelectric Effect in a Superlattice. Superlattices and Microstructures, 37, 87-97. http://dx.doi.org/10.1016/j.spmi.2004.07.003

[33] Mensah, S.Y., Allotey, F.K., Adjepong, S.K. and Mensah, N.G. (1997) Photostimulated Attenuation of Hypersound in Superlattices. Superlattices and Microstructures, 22, 453-457. http://dx.doi.org/10.1006/spmi.1996.0447

[34] Vyazovsky, M.V. and Syrodoev, G.A. (2005) Generation of Acoustic Phonons in Semiconductor Superlattice in the Case of an Intraband Absorption of Electromagnetic Wave. Radiophysics and Quantum Electronics, 48, 390-393. http://dx.doi.org/10.1007/s11141-005-0081-y

[35] Bau, N.Q. and Hieu, N.V. (2014) The Influence of the Electromagnetic Wave on the Quantum Acoustomagnetoelectric Field in a Quantum Well with a Parabolic Potential. Proceedings of PIERS, Guangzhou, 25-28 August 2014.

[36] Shmelev, G.M. and Zung, H. (1977) Fiz. Proceci v Poluprovodnikov. Shttiinca, Kishinev.

[37] Azizyan, A.O. Izv. Akad Nauk. Arm. SSR. Fiz. 9208.

[38] Rabiu, M., Mensah, S.Y., Abukari, S.S., Amekpewu, M., Sefa-Ntiri, B. and Twum, A. (2013) Generation and Propagation of Ultrasonic Waves in Piezoelectric Graphene Nanoribbon. Open Journal of Acoustics, 3, 38-42. http://dx.doi.org/10.4236/oja.2013.33A007 
[39] Dompreh, K.A., Mensah, S.Y., Abukari, S.S., Sam, F. and Mensah, N.G. (2014) Amplification of Acoustic Waves in Graphene Nanoribbon in the Presence of External Electric and Magnetic Field. http://arxiv.org/abs/1410.8064

[40] Dompreh, K.A., Mensah, S.Y., Abukari, S.S., Edziah, R., Mensah, N.G. and Quaye, H.A. (2014) Acoustomagnetoelectric Effect in Graphene Nanoribbon in the Presence of External Electric and Magnetic Field. http://arxiv.org/abs/1412.1678

[41] Nghia, N.V., Bau, N.Q. and Vuong, D.Q. (2012) Calculation of the Acoustomagnetoelectric Field in Rectangular Quantum Wire with an Infinite Potential in the Presence of an External Magnetic Field. Proceedings of PIERS, Kuala Lumpur, 27-30 March 2012, 772-777.

[42] Komirenko, S.V., Kim, K.W., Demidenko, A.A., Kochelap, V.A. and Strocio, M.A. (1999) Cerenkov Generation of High-Frequency Confined Acoustic Phonons in Quantum Wells. http://arxiv.org/abs/cond-mat/9911381

[43] Lee, Y.C. and Tzoar, N. (1969) Phonon Instability in a Magnetic Field. Physical Review, 178, 1303.

[44] Glavin, B.A., Kochelap, V.A., Linik, T.L. and Kim, K.W. (2002) Generation of High-Frequency of Cohenrent Acoustic Phonons in Superlattice under Hopping Transport in Linear Theory of Phonon Instability. Physical Review B, 65, Article ID: 085303.

[45] Ebbecke, J., Strobl, C.J. and Wixforth, A. (2004) Acoustoelectric Current Transport through Single-Walled Carbon Nanotubes. Physical Review B, 70, Article ID: 233401.

[46] Reulet, B., Kasumov, Y.A., Kociak, M., Deblock, R., Khodos, I.I., Gorbatov, Y.B., Volkov, V.T., Journet, C. and Bouchiat, H. (2000) Acoustoelectric Effect in Carbon Nanotubes. Physical Review Letters, 85, 2829-2832.

[47] Zhao, X.F., Zhang, J., Chen, S.M. and Xu, W. (2009) Cerenkov Acoustic-Phonon Emission Generated Electrically from a Polar Semiconductor. Journal of Applied Physics, 105, Article ID: 104514. http://dx.doi.org/10.1063/1.3130400

[48] Nunes, O.A.C. and Fonseca, A.L.A. (2012) Amplification of Hippersound in Graphene under External Direct Current Electric Field. Journal of Applied Physics, 112, Article ID: 043707. http://dx.doi.org/10.1063/1.4748174

[49] Mensah, S.Y., Allotey, F.K.A., Mensah, N.G. and Elloh, V.W. (2003) Amplification of Acoustic Phonons in a Degenerate Semiconductor Superlattice. Physica E, 19, 257-262. http://dx.doi.org/10.1016/S1386-9477(02)01121-9

[50] Mavrinsky, A.V. and Baitinger, E.M. (2009) Thermoelectric Power in Carbon Nanotubes. Semiconductors, 43, $480-484$. 